\title{
Noise-induced phase transitions in field-dependent relaxational dynamics: The Gaussian ansatz
}

\author{
Francisco J. Cao ${ }^{1,2}$ 因 Kevin Wood ${ }^{3,4}$ 男 and Katja Lindenberg团 \\ (1) Departamento de Fúsica Atómica, Molecular y Nuclear, \\ Universidad Complutense de Madrid, Avenida Complutense s/n, E-28040 Madrid, Spain \\ (2) LERMA, Observatoire de Paris, Laboratoire Associé au CNRS UMR 8112, \\ 61, Avenue de l'Observatoire, 75014 Paris, France \\ ${ }^{(3)}$ Department of Chemistry and Biochemistry and Institute for Nonlinear Science, \\ University of California San Diego, 9500 Gilman Drive, La Jolla, CA 92093-0340, USA \\ (4) Department of Physics, University of California San Diego, \\ 9500 Gilman Drive, La Jolla, CA 92093-0340, USA
}

(Dated: October 15, 2018)

\begin{abstract}
We present an analytic mean field theory for relaxational dynamics in spatially extended systems that undergo purely noise-induced phase transitions to ordered states. The theory augments the usual mean field approach with a Gaussian ansatz that yields quantitatively accurate results for strong coupling. We obtain analytic results not only for steady state mean fields and distribution widths, but also for the dynamical approach to a steady state or to collective oscillatory behaviors in multi-field systems. Because the theory yields dynamical information, it can also predict the initialcondition-dependent final state (disordered state, steady or oscillatory ordered state) in multistable arrays.
\end{abstract}

PACS numbers: 05.40.-a,05.10.Gg,64.60.-i

\section{INTRODUCTION}

The interplay of stochasticity and nonlinearity often leads to a wide range of interesting and often counterintuitive behaviors. Noise and nonlinearities have been implicated as sources of both zero-dimensional transitions [1] as well as nonequilibrium phase transitions in spatially extended systems [2]. Particularly intriguing among these is a broad class of macroscopic ordering phenomena that have been called "purely-noise-induced" because order is exhibited only in the presence of noise. These include order-disorder transitions [3, 4, 5], the emergence of periodic spatial structures and pattern formation in increasingly noisy systems [6, 7], and, more recently, purely noise-induced collective oscillatory behavior [8, 9, 10]. Purely noise-induced phase transitions to oscillatory behavior may be dynamically induced and dependent on the Stratonovich drift, i.e., on the interpretation of the noise [3, 9], or it may occur in systems with field dependent relaxational dynamics where the interpretation of the noise at most shifts the transition points [4, 5, [6, 10]. The particular novelty of the latter transition lies in the fact that it arises entirely from an energy functional-like relaxational dynamics, and not from short-time dynamic instabilities which become strengthened and sustained by spatial coupling. As such, these models represent systems that, while decidedly nonequilibrium and potentially time-dependent in nature, nev-

\footnotetext{
*Electronic address: francao@fis.ucm.es

$\dagger$ Electronic address: kwood@ucsd.edu

‡Electronic address: klindenberg@ucsd.edu
}

ertheless maintain certain parallels with equilibrium statistical models, namely the evolution towards the minimum of a (possibly time-dependent) effective potential. Our focus in this paper is on phase transitions in systems with field dependent relaxational dynamics.

Analytic work on these models has been fairly limited, with most of the information coming from numerical simulations. We are not aware of solvable analytic theories for locally coupled (e.g. nearest neighbor) noisy extended systems in which any sort of ordering transition is observed as the system or noise parameters are varied. Globally coupled arrays have been more amenable to mean field theories based on the analytic solution of a Fokker-Planck equation in the stationary state. This approach has been successfully applied to systems that achieve a time-independent steady state. Such mean field theories provide information about the nature of the steady state and the conditions that lead to disordered vs ordered states. Thus, while quite useful for ascertaining asymptotic properties, these static theories provide no insight into the dynamical evolution toward a steady state. Such insight is particularly important in multistable systems.

Most strikingly, these theories fail to provide even a reasonable asymptotic description of phase transitions to collective time-dependent behavior, when the complexity of the corresponding Fokker-Planck equation prohibits a self-consistent analytical treatment [10] even in globally coupled arrays. The complicated behavior seen, for example, in the transition to noise-induced limit cycles thus requires different methods for analytic study [9].

Our purpose here is to provide an approximate analytical method supplementary to the mean field approach for studying the dynamics of noise-induced phase transi- 
tions in relaxational systems inspired by a related quantum field method [11]. By introducing a Gaussian ansatz and a series expansion about the (time-dependent) mean field values, we develop a time-dependent theory which captures the dynamics of these systems in the limit of strong spatial coupling. As well as providing new analytical insight into the evolution to the steady state in one-field systems, this treatment yields a set of simple approximate ordinary differential equations detailing the oscillatory dynamics and the approach to these dynamics in two-field systems. We note that an alternative approach to the one adopted in this paper is to generalize the procedure developed in [9] that gives a set of differential equations for the central moments. It can be shown that this alternative approach gives the same results at leading order for large coupling as the Gaussian ansatz, we are preparing a manuscript where we show the details of the comparison.

The paper is organized as follows. In Sec. II we briefly recall the one- and two-field relaxational models. The mean field equations and the Gaussian ansatz for their solution are presented in Sec. III] In Sec. IV we analyze the one-field system and test our Gaussian ansatz results in the steady state and in the dynamical approach to the steady state, including regimes of multistability. The two-field system is examined in Sec. $[$, where we show that our theory captures the collective oscillatory behavior, including regimes of multistability. In both Secs. IV and $\mathrm{V}$ we explore approximate solutions to the differential equations obtained with the Gaussian ansatz to successfully reduce the problems to quadrature in some regimes. We conclude with a short summary in Sec. VI

\section{THE MODELS}

We briefly recall the one-field and two-field relaxational models. In the one-field case we introduced the Langevin equation defined on a lattice [5],

$$
\dot{\varphi}_{i}=-\Gamma\left(\varphi_{i}\right) \frac{\delta \mathcal{F}(\{\varphi\})}{\delta \varphi_{i}}+\left[\Gamma\left(\varphi_{i}\right)\right]^{1 / 2} \xi_{i}(t),
$$

where $\varphi_{i}$ is the value of the scalar field at site $i, \Gamma\left(\varphi_{i}\right)$ is the field-dependent kinetic coefficient, $\mathcal{F}(\{\varphi\})$ is an energy functional, and $\xi_{i}(t)$ is a spatio-temporal white noise with zero mean and intensity $\sigma^{2},\left\langle\xi_{i}(t) \xi_{j}\left(t^{\prime}\right)\right\rangle=$ $\sigma^{2} \delta_{i j} \delta\left(t-t^{\prime}\right)$. All the quantities introduced previously and those that we introduce later in this paper are consistently adimensionalized. As the choice of noise interpretation does not qualitatively affect the dynamics [5, [6, 10], we choose the Itô interpretation for convenience. The energy functional includes local potentials $V\left(\varphi_{i}\right)$ and a simple harmonic coupling between sites [5, 10]:

$$
\mathcal{F}(\{\varphi\})=\sum_{i=1}^{N}\left[V\left(\varphi_{i}\right)+\frac{K}{4 n} \sum_{\langle i j\rangle}\left(\varphi_{j}-\varphi_{i}\right)^{2}\right] .
$$

Here $N$ is the number of lattice sites and $K$ is the coupling strength. The sum $\sum_{\langle i j\rangle}$ runs over all $n$ sites $j$ coupled to site $i$. For nearest neighbor coupling $n=2 d$ while for global coupling $n=N-1$ (other forms of coupling were also considered [6] but will not be dealt with here). In [5] we carried out comprehensive studies of the phase space diagram in $\left(K, \sigma^{2}\right)$ space where different disordered and ordered phases can be found for different types of local potentials $V(\varphi)$ and of kinetic coefficients $\Gamma(\varphi)$. In general, necessary conditions on these functions for the observation of order-disorder phase transitions include that, at increasingly large $|\varphi|, V(\varphi)$ grow at least harmonically, and $\Gamma(\varphi)$ go to zero so that the effect of the noise is weakened. We were able to analyze the steady states for these systems analytically within a mean field approximation. This approximation leads to a FokkerPlanck equation that can be solved in the steady state, which allowed us to establish the phase space diagram for the steady state, but provided no dynamical information.

The addition of a second field to this problem was introduced in [10],

$$
\begin{aligned}
& \dot{\varphi}_{i}=-\Gamma\left(\varphi_{i}\right) \frac{\delta \mathcal{F}(\{\varphi\})}{\delta \varphi_{i}}+\left[\Gamma\left(\varphi_{i}\right)\right]^{1 / 2} \xi_{i}(t)-\omega z_{i}, \\
& \dot{z}_{i}=\omega \varphi_{i}
\end{aligned}
$$

where $\omega$ is a frequency. This system undergoes a noiseinduced phase transition to collective oscillatory behavior when the noise exceeds a critical intensity. The mean field Fokker-Planck equation for this two-field system can not be solved analytically because it remains time dependent for all time. Thus, all the information obtained up to now has been numerical.

Since our Gaussian ansatz method relies on the mean field evolution equations, in the next section we briefly review the mean field approach and introduce the ansatz.

\section{THE MEAN FIELD AND THE GAUSSIAN ANSATZ}

In the mean field approximation the sum over neighbors connected to site $i$ in the derivative of the energy functional appearing in Eq. (1) or Eq. (3),

$$
\frac{\delta \mathcal{F}\left(\varphi_{i}\right)}{\delta \varphi_{i}}=\frac{\partial V\left(\varphi_{i}\right)}{\partial \varphi_{i}}+K\left(\varphi_{i}-\frac{1}{n} \sum_{\langle i j\rangle} \varphi_{j}\right),
$$

is replaced by the mean field value,

$$
\frac{1}{n} \sum_{\langle i j\rangle} \varphi_{j}(t) \longrightarrow\langle\varphi(t)\rangle \equiv \varphi_{0}(t) .
$$

Since all the sites are then equivalent, the lattice index can be dropped and the set of field equations reduces to a single equation (one-field system),

$$
\dot{\varphi}(t)=a\left(\varphi ; \varphi_{0}(t)\right)+[\Gamma(\varphi)]^{\frac{1}{2}} \xi(t),
$$


or to two coupled equations (two-field system),

$$
\begin{aligned}
& \dot{\varphi}(t)=a\left(\varphi ; \varphi_{0}(t)\right)+[\Gamma(\varphi)]^{\frac{1}{2}} \xi(t)-\omega z, \\
& \dot{z}=\omega \varphi
\end{aligned}
$$

where

$$
a\left(\varphi ; \varphi_{0}(t)\right) \equiv-\Gamma(\varphi)\left\{\frac{\partial V(\varphi)}{\partial \varphi}-K\left[\varphi_{0}(t)-\varphi\right]\right\} .
$$

The unknown mean field $\varphi_{0}(t)$ must be determined selfconsistently,

$$
\varphi_{0}(t)=\langle\varphi(t)\rangle_{\rho} .
$$

Here $\langle\cdot\rangle_{\rho}$ stands for the statistical average with respect to the probability density $\rho$ associated with Eq. (6) or Eq. (7).

The Fokker-Planck equation for the probability density $\rho\left(\varphi, t ; \varphi_{0}(t)\right)$ in the one-field case follows immediately from the Langevin equation (6),

$$
\frac{\partial}{\partial t} \rho=-\frac{\partial}{\partial \varphi}\left[a\left(\varphi ; \varphi_{0}(t)\right) \rho\right]+\frac{\sigma^{2}}{2} \frac{\partial^{2}}{\partial \varphi^{2}}[\Gamma(\varphi) \rho] .
$$

We explicitly note the dependence of $\rho$ on the unknown mean field $\varphi_{0}(t)$, which must be determined via Eq. (9) using the solution of the Fokker-Planck equation. The time dependent probability density has not been found analytically. In the stationary state the left side of Eq. (10) is set to zero and the equation can be solved, to yield the steady state probability density

$$
\begin{aligned}
\rho_{\mathrm{st}}\left(\varphi ; \varphi_{0}\right) & =N\left(\varphi_{0}\right) \Gamma(\varphi)^{-1} e^{-\left(2 / \sigma^{2}\right)\left[V(\varphi)+\frac{K}{2}\left(\varphi_{0}-\varphi\right)^{2}\right]} \\
& =N\left(\varphi_{0}\right) e^{-\left(2 / \sigma^{2}\right) V_{e f f}(\varphi)},
\end{aligned}
$$

where

$$
V_{e f f}(\varphi) \equiv V(\varphi)+\frac{K}{2}\left(\varphi_{0}-\varphi\right)^{2}+\frac{\sigma^{2}}{2} \ln \Gamma(\varphi)
$$

and $N\left(\varphi_{0}\right)$ is the normalization constant. The mean field $\varphi_{0}$ can then be found from Eq. (9). A disordered stationary phase is associated with the solution $\varphi_{0}=0$, while a solution $\varphi_{0} \neq 0$ corresponds to an ordered stationary phase. The phase boundaries for different forms of $V(\varphi)$ and $\Gamma(\varphi)$ are detailed in [5]. In particular, it shows that even if the potential $V(\varphi)$ is monostable (single-well), the presence of field-dependent multiplicative noise leads to an effective bistable (double-well) potential in certain regions of the parameter space. This is precisely the manifestation of a noise-induced phase transition. More elaborate forms of $\Gamma(\varphi)$ could even lead to multistable potentials. Note that the procedure in [5] leads to a complete mean field stationary state analysis, but does not provide information about the dynamics of the approach to the steady state.

For the two-field case the Fokker-Planck equation for the probability density $\rho\left(\varphi, z, t ; \varphi_{0}(t), z_{0}(t)\right)$ follows from the Langevin equation (7),

$$
\begin{aligned}
\frac{\partial}{\partial t} \rho= & -\frac{\partial}{\partial \varphi}\left[\left(a\left(\varphi ; \varphi_{0}(t)\right)-\omega z\right) \rho\right] \\
& -\omega \varphi \frac{\partial}{\partial z} \rho+\frac{\sigma^{2}}{2} \frac{\partial^{2}}{\partial \varphi^{2}}[\Gamma(\varphi) \rho] .
\end{aligned}
$$

This equation has not been solved analytically. Furthermore, since we know from numerical simulations [10] that the system supports collective oscillations, it is necessary to solve the time-dependent problem even to find the long-time behavior. The only available information to date is numerical.

To obtain time-dependent solutions to the one-field and two-field models, we will assume a Gaussian form for the evolving probability density with time-dependent parameters to be found self-consistently from the associated Fokker-Planck equation. Thus, for the one-field problem we take

$$
\rho(\varphi, t)=e^{A\left(\varphi-\varphi_{0}\right)^{2}+C},
$$

where the time dependent mean field $\varphi_{0}(t)$ and inverse width parameter $A(t)$ are to be determined. The parameter $C(t)$ is found from the normalization condition

$$
\int d \varphi \rho(\varphi)=1
$$

to be given by

$$
C(t)=\frac{1}{2} \ln \left(\frac{-A(t)}{\pi}\right) .
$$

It is immediately evident from a comparison of Eqs. (14) and (11) that (14) is at best an approximate solution, but we will subsequently show that for sufficiently strong coupling $K$ this in fact provides an excellent approximation for the mean value and width of the distribution. Note that Eq. (11) implies that in the mean field approximation $A=-V_{e f f}^{\prime \prime}\left(\varphi_{0}\right) / \sigma^{2}$ for the stationary state. For the two-field case we posit the form

$$
\rho(\varphi, z, t)=e^{A\left(\varphi-\varphi_{0}\right)^{2}+E\left(\varphi-\varphi_{0}\right)\left(z-z_{0}\right)+M\left(z-z_{0}\right)^{2}+C},
$$

where now $z_{0}(t), E(t)$ and $M(t)$ are also to be determined. The normalization condition

$$
\int d \varphi d z \rho(\varphi, z, t)=1
$$

fixes $C(t)$,

$$
C=\frac{1}{2} \ln \left(\frac{4 M A-E^{2}}{4 \pi^{2}}\right) .
$$

Note that the Gaussian only has finite norm for $4 M A-$ $E^{2}>0$

We expect these forms to work best for strong coupling. If coupling is too weak, then there is no transition 
to collective behavior and a Gaussian ansatz is not appropriate. As coupling becomes extremely strong, the distribution approaches a $\delta$-function. This provides the motivation for a narrow distribution, whose mean and width we assume to be well captured by a Gaussian when coupling is strong. The Gaussian ansatz thus rests on the observation that $\varphi_{0}(t) \sim z_{0}(t) \sim A\left\langle(\delta \varphi)^{2}\right\rangle \sim E\langle\delta \varphi \delta z\rangle \sim$ $M\left\langle(\delta z)^{2}\right\rangle \sim O\left(K^{0}\right)$ together with the assumption that the second order moments $\left\langle(\delta \varphi)^{2}\right\rangle \sim\left\langle(\delta z)^{2}\right\rangle \sim O(1 / K)$ and the expectation that higher order moments are subleading for large $K$. Here $\delta \varphi \equiv \varphi-\varphi_{0}$ and $\delta z \equiv z-z_{0}$. In the following sections we show that these assumptions lead to consistent results.

\section{SINGLE FIELD}

We start with Eqs. (14) and (16), substitute the Gaussian into the Fokker-Planck equation (10), Taylor expand $a, \Gamma$, and their derivatives, and implement a large- $K$ expansion considering the previous comments. We also recognize that the kinetic coefficient and its derivatives, as well as the local potential and its derivatives, are independent of $K$. The contributions to leading orders in $K$ then result in the set of equations

$$
\begin{aligned}
\dot{A} & =2 A \Gamma_{0}\left(K+\sigma^{2} A\right), \\
\dot{\varphi}_{0} & =-\Gamma_{0} V_{0}^{\prime}-\left(\frac{3 \sigma^{2}}{2}+\frac{K}{A}\right) \Gamma_{0}^{\prime},
\end{aligned}
$$

For each function $f(\varphi)$ we have adopted the notation $f_{0} \equiv f\left(\varphi_{0}\right), f_{0}^{\prime} \equiv[d f(\varphi) / d \varphi]_{\varphi=\varphi_{0}}$, etc. Note that it is clear from these two equations that the system evolves towards $A \sim O(K)$ and $\varphi_{0} \sim O(1)$. We also point out that the normalization condition (16) is consistent with the evolution equation one obtains for $C(t)$, namely, $\dot{C}=\Gamma_{0}\left(K+\sigma^{2} A\right)$. The set of equations (20) and (21) is of course nonlinear and can not be solved exactly analytically, but it is merely a set of two ordinary differential equations whose numerical solution is trivial.

Even so, we can make further analytic progress by noting that for large $K$, and provided the initial value of $A$ is of (negative) $O(K)$, the evolution of $A$ toward its stationary state is clearly faster than that of $\varphi_{0}$, thus allowing us to consider $\Gamma_{0}$ as nearly constant during the relaxation of $A$. In this approximation Eq. (201) is a Riccati equation with solution

$$
A(t)=\frac{-K}{\sigma^{2}} \frac{1}{1-\left(1+\frac{K}{\sigma^{2} A(0)}\right) e^{-2 \Gamma_{0} K t}} .
$$

After the stationary state for $A$ is reached, the evolution of $\varphi_{0}(t)$ is governed by

$$
\dot{\varphi}_{0}=-\Gamma_{0} \frac{d V_{e f f}\left(\varphi_{0}\right)}{d \varphi_{0}} .
$$

Before comparing the results of the theory with those of numerical simulations, we note that in the stationary
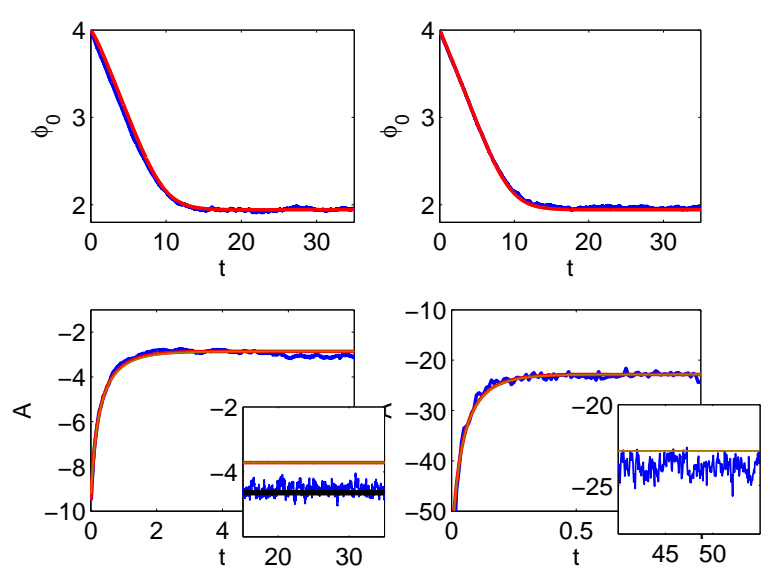

FIG. 1: (Color online) The time evolution of $\varphi_{0}$ (top panels) and $A$ (bottom panels), that give the mean and the inverse width of the distribution, is shown for simulations of globally coupled arrays of $N=4000$ sites and compared to the numerical solution of Eqs. (20) and (21). The left column represents modest coupling, $K=10$, while the right column shows $K=80 ; \sigma^{2}=3.5$ for all plots. The initial values $-A(0)$ are chosen to be $\gtrsim K / \sigma^{2}$; specifically, $A(0)=-9.7$ in the left column and $A(0)=-58$ in the right column. The dark (blue) lines show data from lattice simulations, while the light (red) lines represent theoretical predictions. The very light (brown) curves in the lower right panel and inset represent the uncoupled dynamics given by Eq. (23) and the Riccati equation (22). The lower left inset shows the simulation results and the prediction of the mean field theory (solid black line) in the stationary state, which is exact when $N \rightarrow \infty$. The inset in the bottom right figure shows a close up of the late time evolution. Note the different horizontal and vertical scales in the various panels.

state where $\dot{A}=\dot{\varphi}=0$ we find from Eq. (20) or Eq. (22) that $A=-K / \sigma^{2}$, and that $\varphi_{0}$ obtained from Eq. (21) or (23) is the solution of the condition $d V_{e f f}\left(\varphi_{0}\right) / d \varphi_{0}=0$. The latter is exactly the mean field solution to the problem, which is thus recovered from the Gaussian ansatz. The former differs from the exact inverse width of the distribution (11) by contributions of $O(1)$.

However, the ansatz takes us beyond the stationary solution to provide information about the dynamics of the system as it approaches the steady state. In Fig. 1 1 we show four sets of results for the mean field $\varphi_{0}(t)$ and the inverse width parameter $A(t)$. One is the outcome of the direct simulation of globally coupled arrays for moderate and for strong coupling $K$. The second is the result of integrating Eqs. (20) and (21), the third is the outcome of Eqs. (22) and (23) that assume different relaxation rates, and the fourth is the outcome of the mean field distribution (11). In these and all subsequent figures we have made the representative choices

$$
V(\varphi)=\frac{\varphi^{2}}{2}, \quad \Gamma(\varphi)=\frac{1+\varphi^{2}}{1+\varphi^{4}}
$$

which were also used in our earlier work [ㅁ, $[, 6,10]$. 
The first conclusion is that the time scale of relaxation and the steady state value of $\varphi_{0}$ are correctly predicted by the large coupling theory, even for modest values of $K$. The agreement is spectacular during the approach to steady state. In addition, we note that the time scale separation between $A$ and $\varphi_{0}$ is quite evident for large $K$ and is a reasonable assumption also for modest values, $K \approx 10$. Further discussion of the results in this figure requires that we take note of the different horizontal and vertical scales in the different panels. A small but consistent discrepancy between the Gaussian theory results for $A$ and simulation results for the inverse width of the distribution arises at long times owing to neglected higher order contributions in the theory. In the lower left panel we have expanded the vertical scale to make the difference clear, but note that it is extremely small for large $K$ (see lower right inset). The lower left inset confirms that the simulation and mean field theory stationary widths are in fact identical, as they should be for sufficiently large arrays. While we only show results for two values of $K$ and one lattice size, the results for a range of values of $K(K=20,40,60)$, of lattice sizes $(N=250$ up to $N=16000)$, and of initial conditions in which $A(t=0) \sim-K$ follow the patterns described above. We thus conclude that the Gaussian ansatz theory gives quantitatively excellent results for the evolution of the globally coupled system toward the steady state and for the steady state itself when the coupling is strong and the width of the initial distribution is of the same order as that of the steady state.

Our theory is based on a mean field theory, and so the appropriate comparison with simulations is as we have shown in Fig. 1 with a globally coupled array. However, mean field theories are often used to describe locally coupled systems, and so we compare our theoretical results with simulation results in which the units in a two-dimensional array are connected only to their nearest neighbors. The results are shown in Fig. 2 The theory accurately captures the behavior of $\varphi_{0}$, including both the transient and steady state dynamics, in the large and modest $K$ regimes. However, the inverse width of the distribution (as given indirectly by $A$ ) is underestimated by the theory, as it is by the original mean field theory in this model. A discrepancy of this sort is a ubiquitous feature of mean field theories, which are principally designed to capture the mean field value.

The comparisons so far have relied on the initial value $A(t=0)$ being of (negative) $O(K)$, that is, an initial distribution whose width is of the same order as that of the steady state. When the initial width of the distribution is much larger than that of the steady state, i.e., when $A(t=0)$ is very different from (much smaller in magnitude than) $K$, it becomes more problematic to capture the transient dynamics, although the steady state behavior is still predicted accurately. This is shown in Fig. 3

Finally and importantly, we note that the large coupling theory accurately captures the multistable nature of the dynamics (Fig. (4). This is a new feature of this
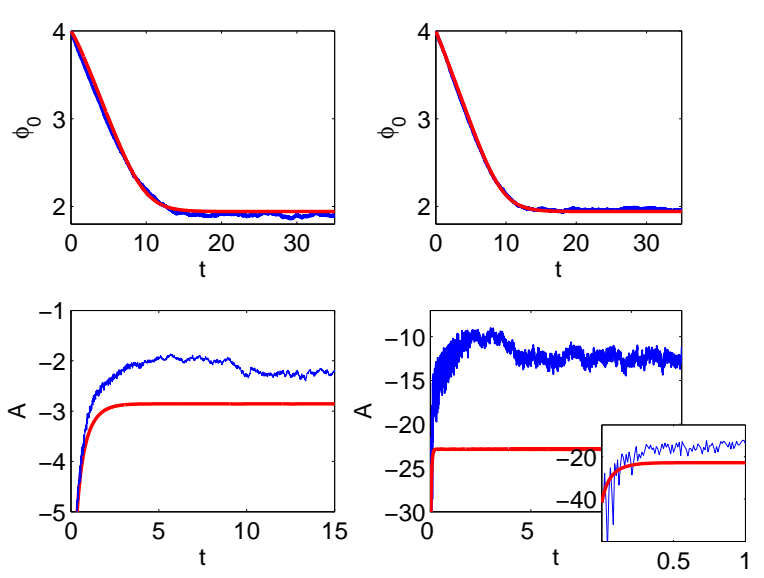

FIG. 2: (Color online) The time evolution of the mean (top panels) and inverse width (bottom panels) is shown for simulations of locally coupled two-dimensional arrays of $N=64 \times 64$ sites and compared to the numerical solution of Eqs. (20) and (21). The left column represents modest coupling, $K=10$, while the right column shows results for $K=80 ; \sigma^{2}=3.5$ for all plots. The dark (blue) lines show data from lattice simulations, while the light (red) lines represent theoretical predictions. The initial values are $A(0)=-9.2$ (left column) and -42 (right column). The inset in the bottom right panel shows a close up of the early time evolution.
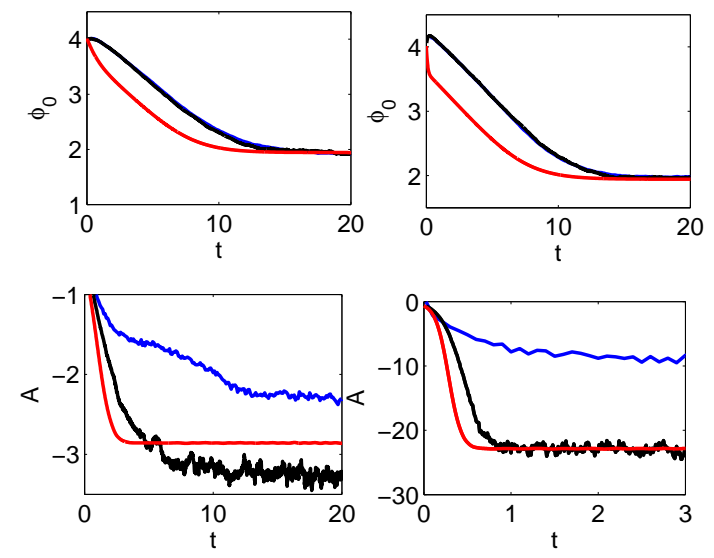

FIG. 3: (Color online) The time evolution of the mean field (top panels) and the inverse width of the distribution (bottom panels) is shown for both locally (dark or blue) and globally (black) coupled simulations and compared to the numerical solution (light or red) of the large $K$ theory. Again, the left column is for $K=10$ and the right for $K=80$. Here we choose $|A(0)|<1$ (specifically, $A(0) \sim-0.1$ ), and the transient dynamics are not well-described for early times. The theory accurately captures the steady-state behavior of the globally coupled simulations in the large $K$ regime. $N=4000$ (globally coupled) and $64 \times 64$ (locally coupled), and $\sigma^{2}=3.5$ for all panels. 

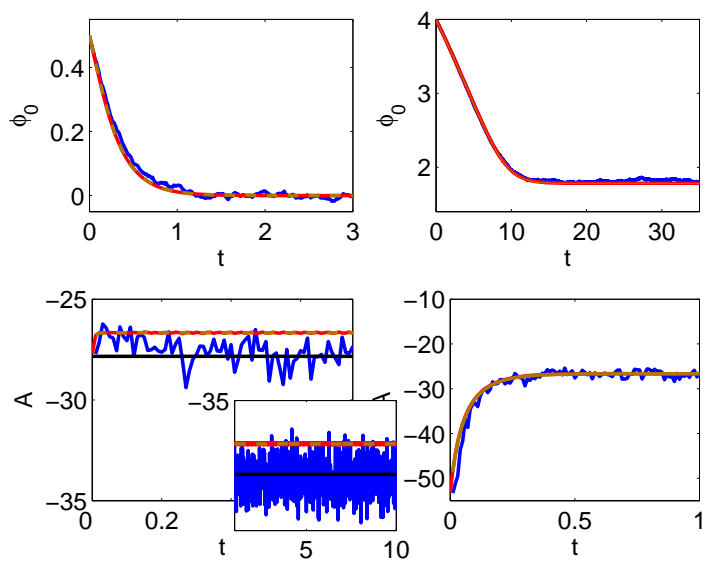

FIG. 4: (Color online) Multistability is captured by the large $K$ theory. The left panel shows the dynamics of the mean field and the inverse width of the distribution for initial conditions leading to a disordered state $\left(\varphi_{0}(0) \approx 0.5, A(0)=-29\right)$. The right panel shows the corresponding plots for initial conditions leading to an ordered phase $\left(\varphi_{0}(0) \approx 4, A(0)=-55\right)$. The inset in the bottom left shows a close up of the steady state behavior. Dark (blue): globally coupled simulations; light (brown): Gaussian ansatz theory; black: mean field theory. In all plots, $K=80, \sigma^{2}=3.0$.

theory that provides dynamical information not provided by the usual mean field theory. In our earlier work we had established this multistability only through direct numerical simulations of the array. For large $K$, the agreement between theory and simulations both in the dynamical regime and in the steady state is quite remarkable for initial conditions leading to the ordered state (right panel). While the mean field theory of course predicts the width of the distribution exactly, the Gaussian ansatz slightly overestimates the width for initial conditions leading to a disordered phase (left panel). The disordered state is marked by a relatively broad distribution, and, not surprisingly, given the underlying requirements stated earlier, the theory does not exactly capture this prediction.

\section{TWO FIELDS}

The more stringent test of the theory lies in the twofield system, where there are only numerical simulation results. Now we begin with Eqs. (17) and (19), substitute the assumed distribution into the two-variable FokkerPlanck equation (13), and again implement a large- $K$ expansion. This results in the set of equations for the coefficients in the Gaussian [13],

$$
\begin{aligned}
\dot{A} & =2 A \Gamma_{0}\left(K+\sigma^{2} A\right), \\
\dot{M} & =\left(\frac{\sigma^{2}}{2} \Gamma_{0} E+\omega\right) E, \\
\dot{E} & =\Gamma_{0}\left(K+2 \sigma^{2} A\right) E+2 \omega(A-M),
\end{aligned}
$$

along with those for the mean values

$$
\begin{aligned}
& \dot{\varphi}_{0}=-\Gamma_{0} V_{0}^{\prime}-\left(\frac{3 \sigma^{2}}{2}+\frac{K}{A}\right) \Gamma_{0}^{\prime}-\omega z_{0}, \\
& \dot{z}_{0}=\omega \varphi_{0} .
\end{aligned}
$$

Again, it is easy to ascertain that the normalization condition (19) is consistent with the evolution equation obtained for $C(t)$.

The solution we are interested in is a collective oscillatory mode, which of course requires the time-dependent solution of the coupled sets of ordinary differential equations (25) and (26), a task which is vastly simpler than the solution of the time-dependent Fokker-Planck equation. However, as in the single field case, we can further simplify the problem of finding the oscillatory long-time behavior of the mean values $\varphi_{0}$ and $z_{0}$ by exploring the regime where the coefficients $A, M$, and $E$ have reached a steady state, that is, by setting the left hand sides in Eq. (25) equal to zero. There are four stationary solutions, one of which is

$$
A=\frac{-K}{\sigma^{2}}, \quad E=0, \quad M=A .
$$

The other solutions, $(A, E, M)=(0,0,0)$, $(A, E, M)=\left(-K / \sigma^{2},-2 \omega /\left(\sigma^{2} \Gamma_{0}\right), 0\right), \quad$ and $(A, E, M)=\left(0,-2 \omega /\left(\sigma^{2} \Gamma_{0}\right),-K / \sigma^{2}\right)$ do not satisfy the condition $4 M A-E^{2}>0$ necessary for proper normalization, cf. Eq. (19), and are hence unphysical.

For the long-time behavior, it now remains to substitute the stationary $A$ into Eq. (26) and solve the coupled set of just two equations,

$$
\begin{aligned}
& \dot{\varphi}_{0}=-\Gamma_{0} \frac{d V_{e f f}\left(\varphi_{0}\right)}{d \varphi_{0}}-\omega z_{0}, \\
& \dot{z}_{0}=\omega \varphi_{0} .
\end{aligned}
$$

We note that this set has one stationary solution, the disordered state $\left(\varphi_{0}, z_{0}\right)=(0,0)$.

We can go even further toward the analytic oscillatory solution by implementing a multiscale perturbation theory [12]. For this purpose we combine (26) into a single second-order differential equation,

$$
\begin{aligned}
& \ddot{z}+\omega^{2} z=\omega G(\varphi), \\
& G(\varphi)=-\Gamma(\varphi) \frac{d V_{\text {eff }}(\varphi)}{d \varphi},
\end{aligned}
$$

with $\varphi \equiv \dot{z} / \omega$. It is understood that the variables are the mean fields and should therefore carry the 0 subscript, which we have omitted for economy of notation. We treat $G$ as a perturbation and write

$$
\ddot{z}+\omega^{2} z=\epsilon \omega G(\dot{z} / \omega),
$$

where $\epsilon$ is a small parameter. The solution is then expressed in terms of different time scales, $\left(T_{0}, T_{1}, \ldots\right)$,

$$
z(t)=Z\left(T_{0}, T_{1}, \cdots\right)=Z_{0}\left(T_{0}, T_{1}\right)+\epsilon Z_{1}\left(T_{0}, T_{1}\right)+O\left(\epsilon^{2}\right),
$$


where $T_{n} \sim O\left(\epsilon^{n} T_{0}\right)$. For the time derivatives we have

$$
\begin{aligned}
\frac{d z}{d t} & =\frac{\partial Z}{\partial T_{0}}+\epsilon \frac{\partial Z}{\partial T_{1}}=\frac{\partial Z_{0}}{\partial T_{0}}+O(\epsilon), \\
\frac{d^{2} z}{d t^{2}} & =\frac{\partial^{2} Z}{\partial T_{0}^{2}}+\epsilon \frac{\partial^{2} Z}{\partial T_{0} \partial T_{1}}+O\left(\epsilon^{2}\right) \\
& =\frac{\partial^{2} Z_{0}}{\partial T_{0}^{2}}+\epsilon \frac{\partial^{2} Z_{1}}{\partial T_{0}^{2}}+\epsilon \frac{\partial^{2} Z_{0}}{\partial T_{0} \partial T_{1}}+O\left(\epsilon^{2}\right)
\end{aligned}
$$

The evolution equation at zero order in $\epsilon$ is

$$
\frac{\partial^{2} Z_{0}}{\partial T_{0}^{2}}+\omega^{2} Z_{0}=0
$$

which has a solution

$$
Z_{0}\left(T_{0}, T_{1}\right)=R\left(T_{1}\right) \cos \left(\omega T_{0}\right)+S\left(T_{1}\right) \sin \left(\omega T_{0}\right) .
$$

The coefficients $R\left(T_{1}\right)$ and $S\left(T_{1}\right)$ are determined by considering the evolution equations at first order in $\epsilon$,

$$
\frac{\partial^{2} Z_{1}}{\partial T_{0}^{2}}+\omega^{2} Z_{1}=-\frac{\partial^{2} Z_{0}}{\partial T_{0} \partial T_{1}}+\omega G\left(\frac{1}{\omega} \frac{\partial Z_{0}}{\partial T_{0}}\right) .
$$

As always in multiscale perturbation theory, in order to avoid secular terms in the solution $R$ and $S$ must be chosen in such a way that resonant terms do not appear in the right hand side of Eq. (35), i.e.,

$$
\begin{aligned}
& \int_{-\infty}^{\infty} d T_{0} \cos \left(\omega T_{0}\right)\left[-\frac{\partial^{2} Z_{0}}{\partial T_{0} \partial T_{1}}+\omega G\left(\frac{1}{\omega} \frac{\partial Z_{0}}{\partial T_{0}}\right)\right]=0 \\
& \int_{-\infty}^{\infty} d T_{0} \sin \left(\omega T_{0}\right)\left[-\frac{\partial^{2} Z_{0}}{\partial T_{0} \partial T_{1}}+\omega G\left(\frac{1}{\omega} \frac{\partial Z_{0}}{\partial T_{0}}\right)\right]=0
\end{aligned}
$$

where from (34) it follows that

$$
\frac{\partial^{2} Z_{0}}{\partial T_{0} \partial T_{1}}=-\frac{\partial R}{\partial T_{1}} \omega \sin \left(\omega T_{0}\right)+\frac{\partial S}{\partial T_{1}} \omega \cos \left(\omega T_{0}\right) .
$$

This immediately leads to the equations for $R$ and $S$,

$$
\begin{gathered}
\frac{\partial R}{\partial T_{1}}=-\frac{\int_{-\infty}^{\infty} d T_{0} \sin \left(\omega T_{0}\right) G\left(\frac{1}{\omega} \frac{\partial Z_{0}}{\partial T_{0}}\right)}{\int_{-\infty}^{\infty} d T_{0} \sin ^{2}\left(\omega T_{0}\right)} \\
\frac{\partial S}{\partial T_{1}}=\frac{\int_{-\infty}^{\infty} d T_{0} \cos \left(\omega T_{0}\right) G\left(\frac{1}{\omega} \frac{\partial Z_{0}}{\partial T_{0}}\right)}{\int_{-\infty}^{\infty} d T_{0} \cos ^{2}\left(\omega T_{0}\right)}
\end{gathered}
$$

The quotients can be computed by introducing a cutoff $\Lambda$ in the integrals, $\int_{-\Lambda}^{\Lambda} d T_{0} \cdots$, and subsequently taking the limit $\Lambda \rightarrow \infty$.

We thus have theoretical predictions at three levels of approximation. The most detailed is the five-equation set (25) and (26). This set of equations contains the dynamical approach to the long time behavior. The second level is contained in Eqs. (27) and (28). This yields the longtime oscillatory behavior of the mean field and the width parameters of the distribution at long times. Finally, Eq. (27) together with (35) and (38) (along with the second equation in (26) ) provide a full long-time solution in terms of quadrature. Note that this explicit solution presents a circular limit cycle of the form $z=S_{0} \sin (\omega t)$ with $S_{0}$ constant $\left[Z_{0}=S_{0} \sin \left(\omega T_{0}\right)\right]$, provided we have $\partial R / \partial T_{1}=0$ [directly satisfied for the choice in (24)] and $\partial S / \partial T_{1}=0$, i.e., if

$$
0=I\left(S_{0}\right)=\int_{-\infty}^{\infty} d T_{0} \cos \left(\omega T_{0}\right) G\left(S_{0} \cos \left(\omega T_{0}\right)\right)
$$

Since our numerical simulations [10] indicate an essentially circular limit cycle near the onset of multistability, we expect that Eq. (39) may provide an accurate prediction of the onset in the strong coupling limit.

We now proceed to test our multi-level theoretical predictions against direct numerical simulations of the array. Again, we adopt the representative choices (24) for the potential and relaxational functions. We concentrate on the multistability onset regime by fixing the value of the noise intensity appropriately.

Figure 5 compares the results of simulations for globally coupled arrays with those of our theory. The agreement is clearly excellent for the limit cycle radius as well as the width parameters for all times. The time scale separation required for the validity of the large $K$ equations is clearly satisfied for $K \sim 80$ and even for modest values, $K \sim 10$. Note that the width parameters reach the steady state very quickly even when $E$ is initially chosen to be of $O(K)$. The theory very slightly overestimates the steady state radius $r \equiv \sqrt{\dot{z}^{2} /\left(2 \omega^{2}\right)+z^{2} / 2}$ for modest $K=10$, and very slightly underestimates the radius for large $K=80$ (neither visible on the scale of the figures). Again as expected, the agreement with the simulations for the locally coupled array is less spectacular (Fig. 6), but the limit cycle radius is still captured very accurately for all times. For visualization purposes, we also show the early time evolution of the theoretical probability distribution function in Fig. 7.

Figure 8 confirms that the phase portrait of the limit cycle from the globally coupled lattice simulations corresponds reasonably well to that predicted by the large $K$ equations, and that the frequency of the oscillations is also accurately predicted. The dynamic evolutions of the simulation and of the theory do not match exactly because the small error in the frequency implies an increasing dephasing with time. The figure shows the case $K=80$, but the results are only representative, and again the theory holds well even for more modest values of $K$ and for a range of initial conditions leading to limit cycle behavior.

Finally, as in the single field problem, the large coupling theory predicts the occurrence of multistable regions in parameter space. In our earlier work [10] we noted the expectation of multistability, but did not explicitly pursue it in our simulations, nor did we have a predictive theory as we now do. In Fig. 9 we show that 

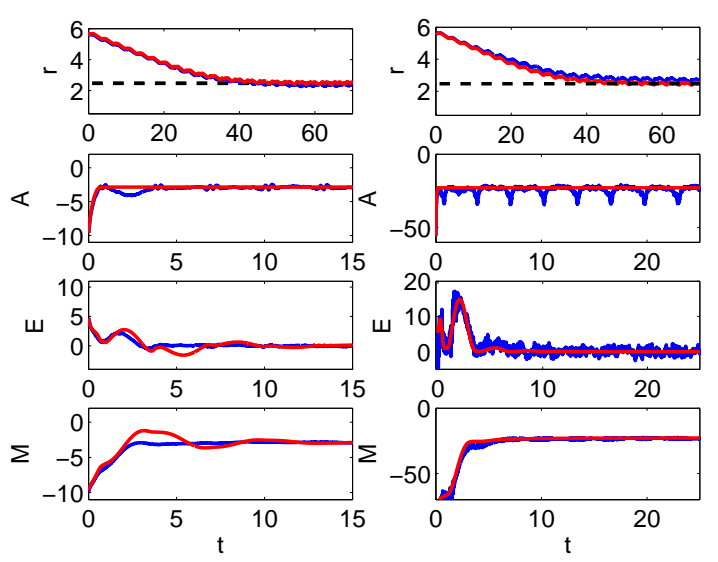

FIG. 5: (Color online) The time evolution of the limit cycle radius, $r$ (top panels) and of the Gaussian ansatz coefficients (three subsequent panels). The dark (blue) curves are appropriate moment results from simulations of globally coupled arrays $(N=4000$ sites), and the light (red) curves are obtained from the solution of Eqs. (25) and (26). $K=10$ in the left column and $K=80$ in the right column. Initial values for left column: $(A, E, M)=(-9.6,4.7,-9.7)$; right column: $(A, E, M)=(-56,7,-79)$. The noise intensity $\sigma^{2}=3.5$ in all panels. The dashed line in the top panels is the steady state radius predicted by multiscale analysis.
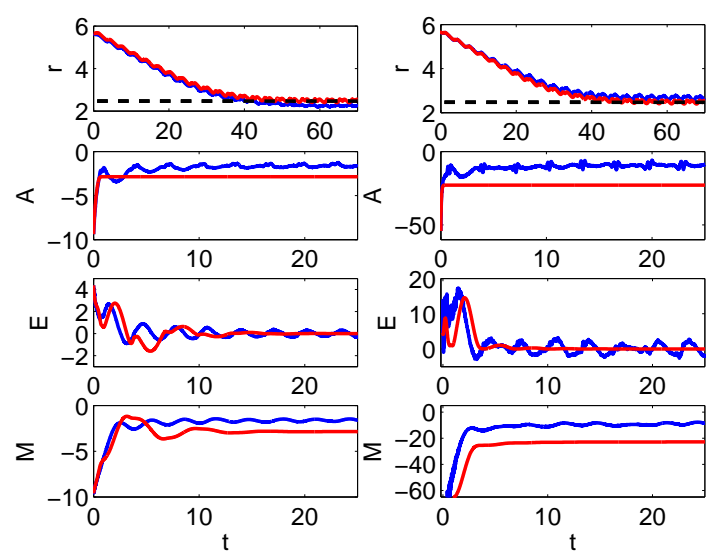

FIG. 6: (Color online) Same as Fig. 5 but now the simulations are for locally coupled arrays. Initial values for left column: $(A, E, M)=(-9.4,4.4,-9.6)$; right column: $(A, E, M)=(-55,5,-76)$.

the multistable nature of the phase transition is fully captured by the large coupling theory. In particular, different initial conditions lead to either a disordered phase or a limit cycle. To test whether the theory correctly predicts the onset of multistability is more cumbersome, but we can at least do it easily in the multiscaling (large $K$ ) regime on the basis of Eq. (39). The value of the noise for onset of multistability in general depends on coupling strength [5, 10], but this dependence greatly weakens with increasing $K$. For example, our simulation

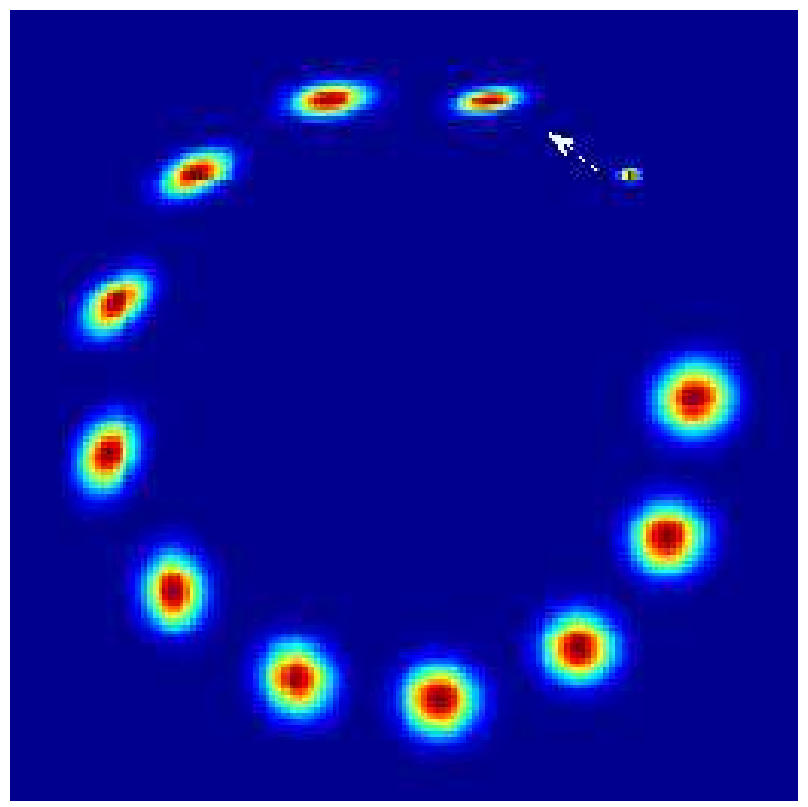

FIG. 7: (Color online) Evolution of the theoretical probability distribution function as obtained by substituting the numerical solution of Eqs. (25) and (26) into Eq. (17). The distribution becomes symmetrical (circular) in $\varphi_{0}$ and $z_{0}$ prior to reaching its steady state radius. The evolution is only shown for the short time leading up to relaxation of the distribution shape. Over longer times, the distribution will continue a circular trajectory whose radius eventually reaches its steady state value (see Fig. 8). $K=10$ and $\sigma^{2}=3.5$.

results indicate that at $K=10$ multistability first occurs at roughly $\sigma^{2} \approx 3$, with the noise value decreasing ever more slowly with increasing $K$ (e.g., $\sigma^{2} \approx 2.6$ for $K=20, \sigma^{2} \approx 2.5$ for $\left.K=40\right)$. At $K=80$ the transition occurs at $\sigma^{2} \approx 2.4$. Equation (39) is in fact independent of $K$. The function $I\left(S_{0}\right)$ is shown explicitly in Fig. 10. The first zero is the disordered state and the second first appears when $\sigma^{2}=2.4$. As noise increases, an intermediate unstable solution also appears, illustrated explicitly in Fig. 9. The multiscaling result thus accurately predicts not only the radius $r=S_{0}$ of the limit cycle but the noise for onset of multistability when coupling is strong, and the increase in the limit cycle radius with increasing noise strength.

\section{CONCLUSIONS}

We have presented an analytical theory for the dynamics of relaxational systems with field dependent coefficients. Based on a Gaussian ansatz and an expansion about the mean field values, we derive ordinary differential equations detailing the time evolution of the field distribution means and widths. In the limit of large coupling, the Gaussian ansatz equations provide a consistent, normalized approximation of the relevant probability distributions which agree with numerical lattice simu- 


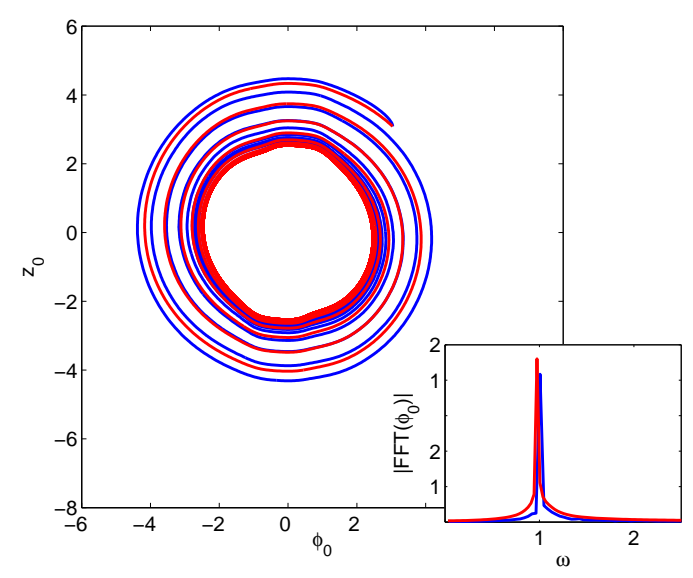

FIG. 8: (Color online) The phase space portraits of lattice simulations (dark or blue) and the large $K$ equations (25) and (26) (light or red) show good agreement in terms of the steady state limit cycle radius, though the theory slights underestimates $r$. Inset: oscillation frequency. $K=80, \sigma^{2}=3.5$, and $\omega=1$.

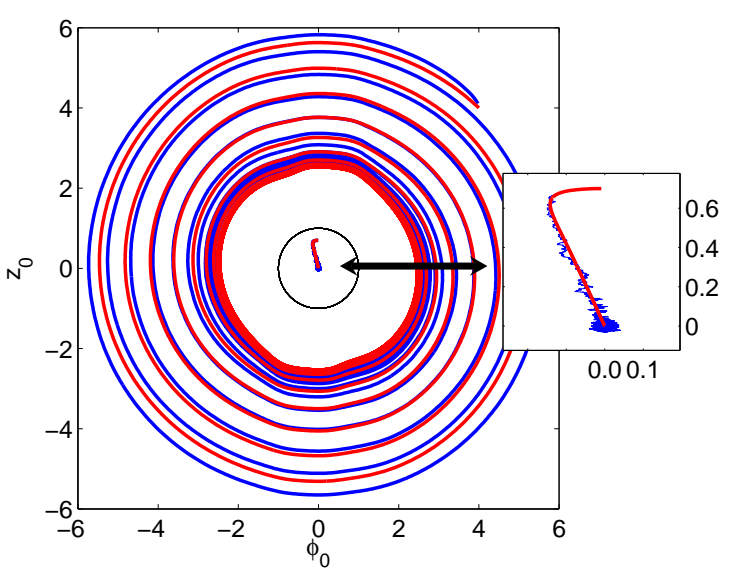

FIG. 9: (Color online) Comparison of the phase space portraits of lattice simulations (dark or blue) and the large $K$ equations (light or red) show that the latter correctly predict multistable behavior. The right inset shows a close up of the indicated portion of the phase portrait. The black curve is the unstable limit cycle obtained from the multiscale analysis. $K=80, \sigma^{2}=3.5$, and $\omega=1$. lations. In particular, our method allows us to study the dynamics of both one- and two-field relaxational systems, including those with oscillatory collective states previously beyond the reach of the static mean field theories. This also provides an analytic access to initial-conditiondependent multistable regimes for noise-induced phase transitions in spatially extended systems.

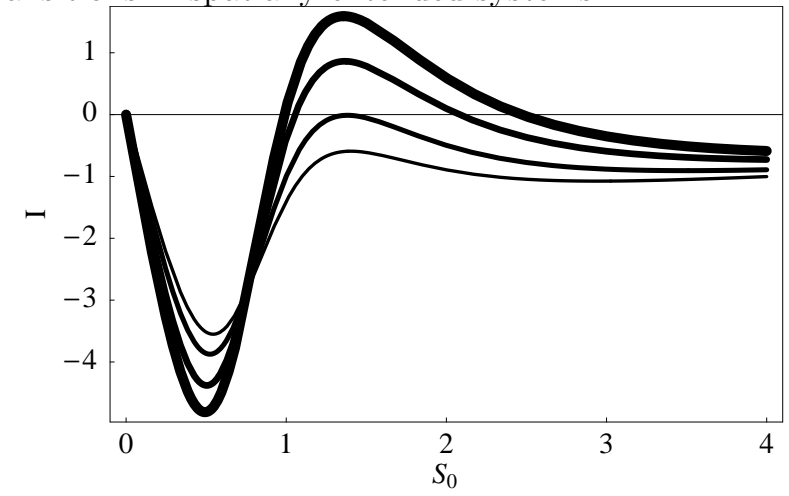

FIG. 10: The multiscale analysis predicts the onset of bistability to occur at $\sigma^{2} \approx 2.4$. Curves are, from thickest to thinnest, $\sigma^{2}=3.5,3.0,2.4,2.0$.

\section{Acknowledgments}

This work was partially supported by the National Science Foundation under Grant No. PHY-0354937, and by the Ministerio de Educación y Ciencia (Spain) under Grants Nos. FIS2006-05895, FIS2005-24376-E, and BFM2003-02547/FISI. F.J.C. also thanks the Universidad Complutense de Madrid for support through the program Profesores Complutenses en el Extranjero, and the Department of Chemistry and Biochemistry of the University of California San Diego for their hospitality.
[1] W. Horsthemke and R. Lefever, Noise-Induced Transitions (Springer, Berlin, 1984).

[2] J. García-Ojalvo and J.M. Sancho. Noise in Spatially Extended Systems (Springer, New York, 1999).

[3] C. Van den Broeck, J. M. R. Parrondo, and R. Toral, Phys. Rev. Lett. 73, 3395 (1994); C. Van den Broeck, J. M. R. Parrondo, R. Toral, and R. Kawai, Phys. Rev. E 55, 4084 (1997).

[4] M. Ibañes, J. García-Ojalvo, R. Toral, and J. M. Sancho, Phys. Rev. Lett. 87, 020601 (2001).
[5] J. Buceta and K. Lindenberg, Phys. Rev. E 69, 011102 (2004).

[6] K. Wood, J. Buceta, and K. Lindenberg, Phys. Rev. E 73, 022101 (2006).

[7] S. E. Mangioni and H. S. Wio, Phys. Rev. E 67, 056616 (2003).

[8] B. von Haeften, G. Izus, S. Mangioni, A. D. Sanchez, and H. S. Wio, Phys. Rev. E 69, 021107 (2004).

[9] R. Kawai, X. Sailer, L. Schimansky-Geier, and C. Van den Broeck, Phys. Rev. E 69, 051104 (2004). 
[10] J. Buceta, K. Wood, and K. Lindenberg, Phys. Rev. E 73, 042101 (2006).

[11] S. Habib, Y. Kluger, E. Mottola, and J.P. Paz, Phys. Rev. Lett. 76, 4660 (1996); F. J. Cao and H. J. de Vega, Phys. Rev. D 63, 045021 (2001); D. Boyanovsky, F.J. Cao, and H. J. de Vega, Nucl. Phys. B 632, 121 (2002); F. J. Cao and H. J. de Vega, Phys. Rev. D 65, 045012 (2002).

[12] L-Y. Chen, N. Goldenfeld, and Y. Oono, Phys. Rev. E $\mathbf{5 4}, 376$ (1996).

[13] Note that if we consider $A \sim M \sim E \sim K$ at leading order, the terms containing $\omega$ will not be retained in Eq. (25). However, at the stationary point $E=0$, so it is interesting to also consider the case when $E \sim K^{0}$ and keep the additional terms that are relevant in this case. Therefore, in Eq. (25) we have considered the possibility that $A \sim M \sim K$ and $E \sim K^{0}$, and have kept the leading order terms for this case. 